\title{
Sélénium et fonction thyroïdienne
}

Le sélénium (Se, oligo-élément important pour l'homme et l'animal [1], fait l'objet d'intenses recherches depuis sa découverte comme constituant de la glutathion peroxydase [2]. Cette enzyme est impliquée dans le système de protection des structures organiques contre les effets toxiques des radicaux libres. Ainsi une carence en Se pourrait affecter la capacité de défense anti-oxydante de l'organisme, et jouer un rôle important dans la pathogénie de nombreuses maladies [3]. Actuellement, cette enzyme ne constitue plus l'unique sélénoprotéine connue [47]. La découverte de l'implication du Se dans l'activité d'une autre enzyme, la 5 '-désiodase type I (5'-DI) [8], a suscité un intérêt particulier du fait de l'importance physiologique de cette enzyme dans le métabolisme des hormones thyroïdiennes.

\section{Sélénium et 5'-désiodase type I}

La 5'-DI, enzyme catalysant la conversion de la $3,3^{\prime}, 5,5^{\prime}$-tétraiodothyronine (T4) en 3,3',5-triiodothyronine (T3), notamment dans la thyroïde, le foie et le rein, fournit la plupart de la T3 circulante d'origine hépatique [9]. Il y a quelques années, un lien fut établi entre l'enzyme 5'-DI et le sélénium [8]. Par la suite, la 5'-DI fut identifiée comme une sélénoenzyme [9]. De surcroît, on a montré que le sélénium se trouve dans le site actif de la 5'-DI sous forme de résidu sélénocystéine codé au niveau de l'ARN messager par un triplet UGA [7, 11]. Cela est confirmé par plusieurs travaux revus par Berry et Larsen [12] qui précisent que la 5'-DI est également une sélénoenzyme contenant de la sélénocystéine chez l'homme. Il n'est donc pas surprenant qu'un régime alimentaire appauvri en sélénium réduise fortement l'activité de la 5'-DI hépatique chez
Toutefois, il a été démontré que les besoins en Se pour une activité normale de la 5'-DI chez le rat, sont beaucoup plus faibles pour la thyroïde $(0,01 \mathrm{mg}$ de $\mathrm{Se} / \mathrm{kg}$ d'aliment $)$ que pour le foie $(0,05 \mathrm{mg}$ de $\mathrm{Se} / \mathrm{kg}$ d'aliment) [14] et qu'une carence en Se réduit fortement l'activité de la 5'-DI dans le foie, alors qu'elle ne la change pratiquement pas dans la thyroïde $[14,15]$. Ainsi, lors d'un apport insuffisant en Se, la glande thyroïde semble être prioritaire pour le Se, par rapport au foie, et capable de retenir cet oligoélément en quantité suffisante pour la synthèse de la 5'-DI. D'autres travaux plus récents semblent aussi être en faveur de cette hypothèse [16, 17]. Outre cette spécificité tissulaire dans la préservation du sélénium, il existe aussi une incorporation intracellulaire préférentielle du sélénium dans la 5'-DI par rapport à l'autre sélénoenzyme, la glutathion peroxydase [18].

\section{Sélénium \\ et autres enzymes thyroïdiennes}

Partant de la découverte de la nouvelle fonction du sélénium dans la 5'DI, la présence de cet oligoélément a été également recherchée dans d'autres enzymes thyroïdiennes aussi importantes, telles que la 5'-désiodase type II (5'-DII) et la 5-désiodase type III (5-DIII) [18] : (1) la 5'-DII, qui permet la conversion de $\mathrm{T} 4$ en T3, notamment dans le cerveau, l'hypophyse et le tissu adipeux brun, n'est pas une sélénoenzyme [18], quoique son activité se trouve abaissée dans les conditions de carence en sélénium [17, 20]. Cette baisse serait plutôt due à un effet direct de l'augmentation de T4 sérique observée dans ces conditions puisque l'activité de la 5'-DII varie inversement avec les concentrations sériques de T4. On estime donc qu'une telle réduction est une réponse protectrice pour le cerveau, permettant le maintien d'une concentration normale de T3 tissulaire en dépit de l'élévation de T4 circulante [20]. (2) La 5-DIII (enzyme rendant T4 et T3 inactives par désiodation), quant à elle, est aussi une sélénoenzyme contenant de la sélénocystéine, et son absence de modification d'activité dans les conditions de carence en sélénium, rapportée par certains auteurs [21, 22], pourrait être une simple conséquence de la prédilection de cet élément pour certains tissus dont le cerveau forme un bon exemple [18].

\section{Sélénium et dysthyroïdie}

En 1984, Aihara et al. [23] furent les premiers à rapporter une diminution du Se plasmatique, ainsi qu'une corrélation négative entre les valeurs sériques de $\mathrm{T} 4$ et $\mathrm{T} 3$ et la concentration plasmatique du Se chez des malades hyperthyroïdiens comparés à des sujets témoins. Selon ces auteurs, il est possible que la diminution du Se plasmatique corresponde à une augmentation des besoins en Se dans certains tissus comme le foie, les érythrocytes et le cœur, et qu'elle ne soit pas en relation avec l'état des réserves. Pour notre part, nous avons observé que le Se augmente significativement dans le sang total chez. des patients hyperthyroïdiens marocains comparés à des sujets témoins (Hmidi et al., soumis pour publication).

Dans les situations d'hypothyroïdie, Vanderpas et al. [24] ont noté une forte déficience en Se associée à une réduction concomitante de l'activité de la sélénoenzyme glutathion peroxydase chez des sujets atteints de crétinisme hypothyroïdien. Ces auteurs signalent aussi une relation géographique entre cette affection et la déficience en Se en Afrique centrale (Zaire). Toutefois, des essais cliniques ont révélé que l'administration du Se seul 
Tableau I

EFFET D'UN DÉFICIT EN SÉLÉNIUM SUR LA FONCTION THYROÏDIENNE CHEZ DIFFÉRENTES ESPĖCES

\begin{tabular}{|c|c|c|c|c|c|c|}
\hline & Espèce & Plasma & Foie & Thyroïde & Cerveau & Hypophyse \\
\hline TSH & Homme & $\leftrightarrow \quad[28]$ & & & & \\
\hline & Rat & $\leftrightarrow \quad[17]$ & & & & \\
\hline \multirow[t]{3}{*}{ T4 } & Homme & $\uparrow[28]$ & & & & \\
\hline & Rat & {$[8,14,17]$} & $\uparrow[17]$ & $\downarrow[17]$ & & \\
\hline & Cobaye & $\leftrightarrow[30]$ & $\leftrightarrow[30]$ & & & \\
\hline \multirow[t]{4}{*}{ T3 } & Homme & $\leftrightarrow\left[\begin{array}{l}{[28]} \\
\downarrow\end{array}\right.$ & $\downarrow$ & $\mid$ & & \\
\hline & ndit & $\leftrightarrow[14]$ & $\downarrow[1 /]$ & & & \\
\hline & Veau & {$[29]$} & & & & \\
\hline & \multicolumn{4}{|c|}{ Activité enzymatique } & & \\
\hline 5'désiodase & Rat & & $\downarrow[8,14,17]$ & $\leftrightarrow[14,15]$ & & \\
\hline type I & Cobaye & & $\downarrow[30]$ & & & \\
\hline $\begin{array}{l}5^{\prime} \text { désiodase } \\
\text { type II }\end{array}$ & Rat & & & & $\downarrow[20]$ & $\downarrow[17]$ \\
\hline $\begin{array}{l}5^{\prime} \text { désiodase } \\
\text { type III }\end{array}$ & Rat & & & & $\leftrightarrow \quad[22]$ & \\
\hline Glutathion & Rat & & $\downarrow[8,14,17]$ & $\downarrow[14]$ & & \\
\hline Poids de la thyroïde & Rat & & & $\begin{array}{l}\uparrow[17] \\
\leftrightarrow[14]\end{array}$ & & \\
\hline
\end{tabular}

$\leftrightarrow$ Aucun changement; $\downarrow$ diminution; $\uparrow$ augmentation; I références.

pendant deux mois à des sujets présentant un syndrome de crétinisme, avant l'amélioration de leur statut en iode, ne corrige pas les paramètres thyroïdiens mais, au contraire, aggrave la fonction thyroïdienne initialement affectée chez ces patients [25]. En eff et, après deux mois de supplémentation, la concentration sérique du Se augmente et atteint des valeurs physiologiques; en revanche, la valeur moyenne de T4 sérique diminue largement. Quant à celle de TSH, elle augmente significativement. Tout se passe donc comme si la déficience en Se observée chez ces sujets hypothyroïdiens modérait la sévérité de l'hypothyroïdie et protégeait contre les effets de la carence en iode. Elle pourrait aussi protéger le développement du cerveau du fotus contre la baisse de T4 par réduction de la désiodation périphérique de T4 maternelle qui constitue la principale source de T3 cérébrale durant les premiers mois de grossesse. En revanche, la carence combinée en sélénium et en iode pourrait favoriser les processus dégénératifs de la glande thyroïde aboutissant au crétinisme endémique myxœdémateux. Des études épidémiologiques et expérimentales plus récentes confirment ces résultats [26, 27]. C'est pour cette raison qu'on a conclu que l'apport de sélénium seul ne peut être réalisé sans l'administration concomitante de l'iode chez une population carencée à la fois en iode et en sélénium. En revanche, si les réserves en iode sont restaurées d'abord, et le sélénium administré par la suite, la normalisation de la synthèse, de l'activité et du métabolisme des hormones thyroïdiennes semble alors possible [22].

L'altération du statut thyroïdien, traduite par une valeur de T4 circulante élevée alors que celle de T3 est légèrement abaissée ou pratiquement inchangée, observée dans les conditions de carence nutritionnelle en sélénium chez différents animaux, témoigne aussi de l'importance de cet oligoélément dans la fonction thyroïdienne [14, 20, 28, 29]. A l'exception de la T4, le métabolisme des hormones thyroïdiennes est aussi altéré par la carence en sélénium chez le cobaye [30].
Au total, l'ensemble de ces recherches converge vers l'idée que l'effet sur la fonction thyroïdienne d'une carence en Se pourrait se traduire par l'altération, plus ou moins importante suivant les tissus, de l'activité de deux sélénoenzymes: la 5'-DI, et la glutathion peroxydase. Une perturbation de l'activité de la 5'-DII, semble aussi contribuer à cet effet [17] (Tableau I). Il est évident que ce tableau n'explique certainement pas tout et nécessite d'autres recherches. En effet, l'interaction entre le Se et la fonction thyroïdienne est beaucoup plus complexe qu'un simple effet sur la conversion tissulaire de T4 en T3 et l'apparition d'un état d'hypothyroïdie [31]. Récemment, Wu et al. [15] ont suggéré que la réduction de l'activité de la 5'DI résultant normalement de la carence en sélénium, est associée à une orientation importante de $\mathrm{T} 4$ et $\mathrm{T} 3$ dans la voie métabolique de sulfatation. Par ailleurs, notre équipe a entamé récemment une étude de l'influence du statut des hormones thyroïdiennes sur la distribution tissulaire du Se chez le rat, notamment par 
la technique d'autoradiographie, dont les principaux aspects sont décrits par Bénard et al. [32]. Nos premiers résultats reflètent une forte perturbation dans la distribution tissulaire du Se en fonction du statut des hormones thyroïdiennes (Bénard et al., en préparation).

\section{Conclusion}

On dispose de plus en plus d'éléments permettant d'attribuer au Se un rôle déterminant dans la fonction thyroïdienne. Il est donc intéressant de poursuivre des recherches pour mieux comprendre ce rapport et préciser l'importance clinique du Se dans les maladies thyroïdiennes. Ces études pourraient déboucher sur des conseils nutritionnels pour prévenir et traiter certaines maladies thyroïdiennes

\section{Najah Hmidi}

Enseignant chercheur, laboratoire de physiologie animale, Faculté des sciences, Rabat, Maroc.

\section{Mimoun Kessabi}

Professeur, chef de département de toxicologie et pharmacie, Institut Agronomique et Vétérinaire Hassan II, Rabat, Maroc.

\section{Patrick Bénard}

Professeur, laboratoire de radioéléments et d'études métaboliques, École nationale Vétérinaire, 23, chemin des Capelles, 31300 Toulouse, France.

\section{Hassan Lachkar}

Professeur, service de médecine E, hôpital Avicenne, Rabat, Maroc.

\section{Ahmed Hamliri}

Professeur, laboratoire de Toxicologie, Ins titut Agronomique et Vétérinaire Hassan II, Rabat, Maroc.

\section{Wail Benjelloun}

Professeur, vice-président, Université Al Akhawayn, Ifrane, Maroc.

\section{TIRÉS À PART}

\section{RÉFÉRENCES}

1. Underwood EJ. Selenium. In: Trace elements in human and animal nutrition, 4th ed. New York: Academic Press, 1997 : 302-46.

2. Flohe L, Günzler WA, Schock HH. Glutathione peroxydase: a selenoenzyme. FEBS Lett 1973; 32: 132-4.

3. Diplock AT. Antioxidant nutrients and disease prevention: an overview. Am J Clin Nutr 1991 ; 53 : 189S-93.

4. Read R, Yang JG, Hill KE, Burk RF. Characterization of rat selenoprotein $P$ : the major form of serum selenium in rats. FASEB J 1990; 4 : A371.

5. Burk RF. Molecular biology of selenium with implications for its metabolism. FASEB $J 1991$; 5: 2274-9.

6. Zachara BA. Mammalian selenoproteins. Trace Elem Electrolytes Health Dis 1992; 6 : $137-51$.

7. Sturchler-Pierrat C Carbon P, Krol A Sélénium, sélénoprotéines: une autre lecture du code génétique. médecine/sciences 1995; 11 : 1081-8.

8. Beckett GI, Beddows SE, Morrice PG, Nicol F, Arthur JR. Inhibition of hepatic deiodination of thyroxine is caused by selenium deficiency in rats. Biochem $J$ 1987; $248:$ 443-7.

9. Pilo A, Iervasi G, Vitek F, Ferdeghini M, Cazzuola $F$, Bianchi $R$. Thyroidal and peripheral production of 3,5,3'-triiodothyronine in humans by multicompartmental analysis. Am J Physiol 1990; 258: E715-26.

10. Behne D, Kyriakopoulos A, Meinhold H, Köhrle J. Identification of type I iodothyronine 5'-deiodinase as a selenoenzyme. Biochem Biophys Res Commun 1990; 173: 1143-9.

11. Berry MJ, Banu L, Larsen PR. Type I iodothyronine deiodinase is a selenocysteine-containing enzyme. Nature 1991; 349: 438-40.

12. Berry MJ, Larsen PR. The role of selenium in thyroid hormone action. Endocrinol Rev 1992; 13: 207-19.

13. Vadhanavikit S, Ganther HE. Effects of selenium depletion and repletion on hepatic 5'-deiodinase (type I), glutathione-Stransferase, and glutathione peroxydase in the rat. FASEB J 1990; 4: A371.

14. Vadhanavikit S, Ganther HE. Selenium requirements of rats for normal hepatic and thyroidal 5'-deiodinase (type I) activities. $I$ Nutr 1993; 123: 11248.

15. Wu SY, Huang WS, Chopra IJ, Jordan M, Alvarez D, Santini F. Sulfation pathway of thyroid hormone metabolism in seleniumdeficient male rats. Am J Physiol 1995; 268 . E572-9.

16. Christensen MJ, Cammack PM, Zwahlen BA, Wray CD. Tissue specificity of selenoprotein gene expression in rats fed three levels of dietary selenium. FASEB J 1993; 7: A277.

17. Beckett GJ, Nicol F, Rae PWH, Beech S, Guo Y, Arthur JR. Effects of combined iodine and selenium deficiency on thyroid hormone metabolism in rats. Am J Clin Nutr $1993 ; 57$ (suppl) : 240S-3

18. Larsen PR, Berry MJ. Nutritional and hormonal regulation of thyroid hormone deiodinases. Annu Rev Nutr 1995; 15: 323-52.

19. Safran M, Farwell AP, Leonard JL. Evidence that type II $5^{\prime}$-deiodinase is not a selenoprotein. I Biol Chem 1991; 266 : 13477-80.

20. Chanoine JP, Safran M, Farwell AP, et al Selenium deficiency and type II 5'-deiodinase regulation in the euthyroid and hypothyroid rat: evidence of a direct effect of thyroxine. Endocrinology $1992 ; 131: 479-84$.

21. Chanoine JP, Alex S, Stone S, et al. Placental 5-deiodinase activity and fetal thyroid hormone economy are unaffected by selenium deficiency in the rat. Pediatr Res 1993 34: 288-92.

22. Köhrle J. Thyroid hormone deiodination in target tissues - a regulatory role for the trace element selenium? Exp Clin Endo crinol 1994; 102 : 63-89.

23. Aihara K, Nishi $\mathrm{Y}$, Hatano $\mathrm{S}$, et al. Zinc, Copper, manganese, and selenium metabolism in thyroid disease. Am J Clin Nutr 1984; 40: 26-35.

24. Vanderpas JB, Contempré B, Duale NL, et al. Iodine and selenium deficiency associated with cretinism in northern Zaire. $A m$ J Clin Nutr 1990; 52: 1087-93.

25. Contempré B, Dumont JE, Ngo Bebe, Thylly CH, Diplock AT, Vanderpas J. Effect of selenium supplementation in hypothyroid subjects of an iodine and selenium deficient area: the possible danger of indiscriminate supplementation of iodine-deficient subjects with selenium. I Clin Endocrinol Metab 1991; 73 : 213-5.

26. Corvilain B, Contempré B, Longombé $\mathrm{AO}$, et al. Selenium and the thyroid: how the relationship was established. Am J Clin Nutr 1993; 57 (suppl) : 244S-8S.

27. Vanderpas JB, Contempré B, Duale NL, et al. Selenium deficiency mitigates hypothyroxinemia in iodine-deficient subjects. $A m$ J Clin Nutr 1993; 57 (suppl) : 271S-5.

28. Terwolbeck K, Behne D, Meinhold $\mathrm{H}$, Menzel H, Lombeck I. Increased plasma T4 levels in children with low selenium state due to reduced type I iodothyronine 5'deiodinase activity? I Trace Elem Electrolytes Health Dis 1993; 7: 53-5.

29. Arthur JR, Morrice PC, Beckett GJ. Thyroid hormone concentrations in selenium deficient and selenium sufficient cattle. Res Vet Sci $1988 ; 45$ : 122-3.

30. Cammack PM, Zwahlen BA, Cristensen MJ. Selenium deficiency alters thyroid hormone metabolism in guinea pigs. $J$ Nutr $1995 ; 125: 302-8$.

31. Arthur JR, Nicol F, Beckett GJ. Selenium deficiency, thyroid hormone metabolism, and thyroid hormone deiodinases. $A m J$ Clin Nutr 1993; 57 (suppl) : 236S-9.

32. Bénard P, Burgat V, Rico AG. Application of whole body autoradiography in toxicology. CRC Crit Rev Toxicol $1985 ; 15 ; 181-215$. 